Review article

Central Eur J Paed 2018;14(1):22-26

DOI $10.5457 / \mathrm{p} 2005-114.195$

\title{
The current role of skeletal survey in children with suspected skeletal dysplasia
}

\author{
Damjana Ključevšek \\ University Clinical Centre, Children's \\ Hospital Ljubljana, Radiology Unit
}

Correspondence:
damjana.kljucevsek@gmail.com
Tel.: + 38615229264
Fax.: + 38615222993

Received: October 14, 2017

Accepted: November 3, 2017

Key words: Skeletal dysplasia - Radiology Skeletal survey.

\begin{abstract}
The purpose of this mini review is to emphasize the importance of skeletal survey, its position in the diagnostic algorithm of skeletal dysplasia (SD), and the importance of its systematic analysis. Skeletal survey, the series of plain radiographic bone imaging, is still the first line imaging method in children with suspected SD, despite fast developing gene and biochemical methods. SDs are a genetically heterogeneous group of disorders affecting skeletal development, with a wide clinical spectrum. The diagnosis of a SD is usually based on a combination of clinical, radiological, morphological, and lately biochemical and molecular studies, which enable more extensive insight into the genes controlling normal skeletal development and lead to more adequate genetic counselling. Standardised imaging protocols of skeletal survey help radiologists to plan and optimize an appropriate imaging series, reduce radiation dose from unnecessary exposure, and facilitate earlier diagnosis. Since 1970 the International Nomenclature of Constitutional Diseases of Bones has been developed and updated at regular intervals (last reclassification in 2015). Reclassifications are based on new insights into gene and/or protein, molecular defects. A multidisciplinary approach is necessary due to the considerable heterogeneity. An accurate diagnosis is essential for proper management of the affected individual and for genetic counselling. Various endocrine and metabolic diseases affecting bones should be considered in differential diagnosis. Conclusion - A systemic approach to skeletal survey imaging analysis in children with suspected SD is crucial to avoid missing important details regarding the morphological or structural features of the bones, which may lead to a false diagnosis of SD.
\end{abstract}

\section{Introduction}

Skeletal dysplasia (SD) is a large, heterogeneous group of inherited disorders of cartilage/ bones that affect their growth, morphology and integrity. They can be classified as osteochondrodysplasias and dysostoses (1). Osteochondrodysplasias are conditions associated with abnormalities of the growth (dysplasia) or texture (osteodystrophy) of the bone/cartilage due to genetic mutations. The changes in the affected bones may progress throughout life, changing their phenotype. Dysostoses are conditions secondary to abnormal blastogenesis in utero in the first 6 weeks of foetal life. They remain static throughout life. Currently more than 400 different entities have been described based on radiological, molecular and biochemical criteria. They may manifest in ways ranging from a barely noticeable abnormality to a severe and lethal condition. An overall prevalence of SD 2.3-7.6 per 10.000 births has been reported in different epidemiologic studies $(2,3)$. 
The "International Nomenclature of Constitutional Diseases of Bones" group has classified these disorders intermittently since its first publication in 1970. In the 1970s, the categories were purely clinical and descriptive. The study of the genetics of SDs led to a new nomenclature based on new findings, primarily molecular in nature. Therefore, the combination of clinical, radiological and molecular knowledge has become the basis for new classifications $(4,5)$. Forty-two groups and 436 disorders were classified in the latest 2015 revision of the Nosology and Classification of Genetic Skeletal Disorders (6). Families of disorders that share a common molecular basis or pathway were created. In addition, recent findings have demonstrated that identical phenotypes may result from mutations in different genes that act through a similar pathway, and that the same gene can cause different phenotypes.

It is important to remember that an accurate diagnosis of a SD is still based on detailed evaluation of clinical (family history, physical examination) and radiographic findings, followed by molecular and the biochemical tests. In general, the SDs' common clinical and radiological findings help us to group them in several ways regardless of the specific diagnosis. Individuals with SD have to be identified, because they present with significant morbidities due to the destruction of bone and cartilage caused by defects in growth, bone modelling and regeneration, and other comorbidities (affecting the liver, kidney, lung and brain). The most widely used method for differentiating skeletal disorders showing different skeletal morphological and structural abnormalities, is "oldfashioned" radiography.

In this mini review we will emphasize the importance of skeletal survey, its position in the diagnostic algorithm of SD, and the importance of its systematic analysis.

\section{A diagnostic approach to suspected skeletal dysplasia}

Some forms of skeletal dysplasia may be recognized in utero by identifying a skeletal abnormality on antenatal ultrasound. After birth, SD is suspected in children with unexplained short statue, particularly if it is disproportionate, and in children with particularly facial dysmorphism. Dysplasia should be differentiated from other causes of short stature, such as familial short stature, endocrine disorders and metabolic disorders. Clinical evaluation includes various anthropometric measurements, description of the limb involvement, hair quality, cleft palate, eye abnormalities, immunological/haematological data, and even internal organ abnormalities, such as cystic kidney, or hepatosplenomegaly seen during abdominal ultrasonography.

A radiological evaluation is an integral part of the post-natal diagnostic workup of SD. A skeletal survey, composed of a series of radiographs, is performed in a child with a suspected bone disorder. The skeletal survey includes radiography of the skull in 2 views, the thoracolumbar spine in 2 views, the chest, pelvis, one upper limb, one lower limb, and the left hand (including assessment of bone age) (7). In new-borns, due to the small size of the baby, images can usually be joined into an $\mathrm{AP}$ and a lateral babygram, with all the upper and lower extremities (Fig.1 and Fig. 2) (8).

Sometimes dedicated views of specific sites may be required for adequate bone visualisation and assessment. It is important to keep in mind that a complete skeletal survey is not necessary in patients with proportionate short stature or the presence of specific local skeletal abnormalities, according to the published guidelines (7). This protects children from unnecessary radiation exposure. In general, there is nothing to be gained from repeating the images; imaging should not be repeated more than once or twice, over a period of less than one year. It is also not 


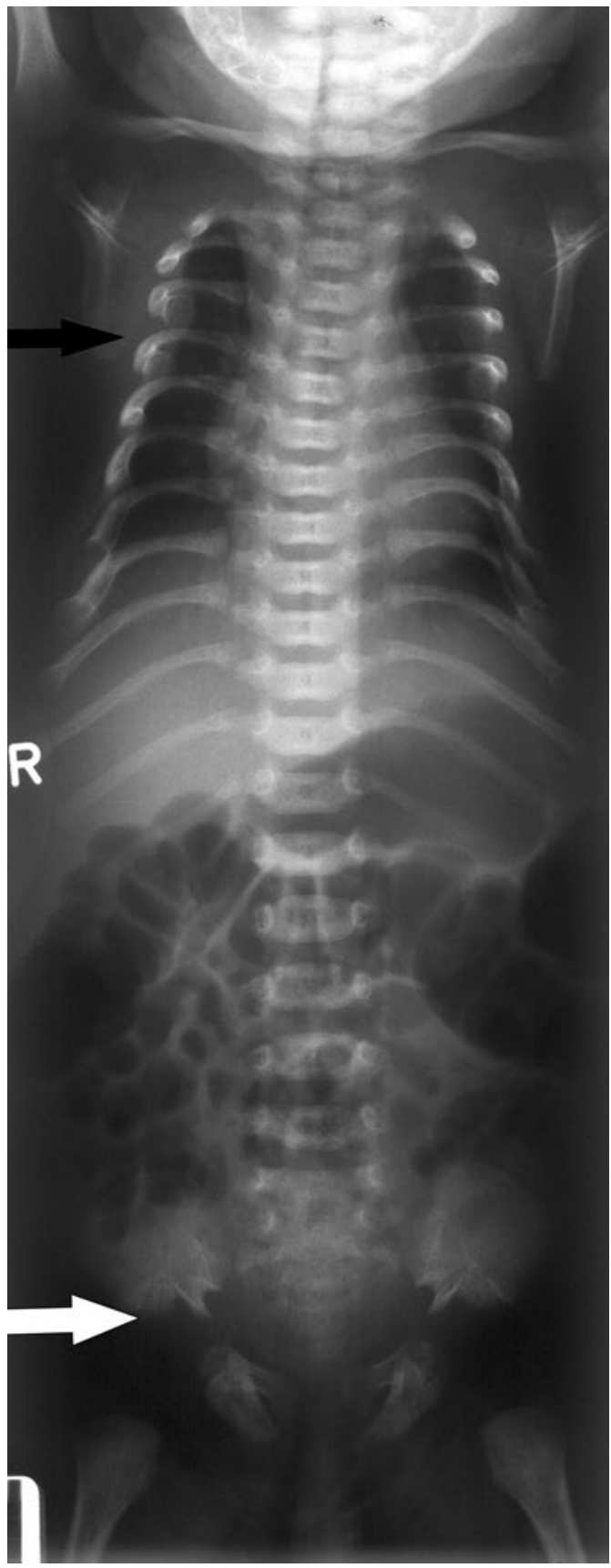

Fig. 1. "Babygram" of a new-born girl with asphyxiating thoracic dystrophy (Jeune syndrome): long and barrel-shaped thorax, handlebar clavicles, short horizontal ribs with bulbous anterior ends, and normal spine. Morphologically altered pelvis with small, flared iliac wings, narrowed sacrosciatic notches, and dysplastic trident acetabular roof (from our own archives).

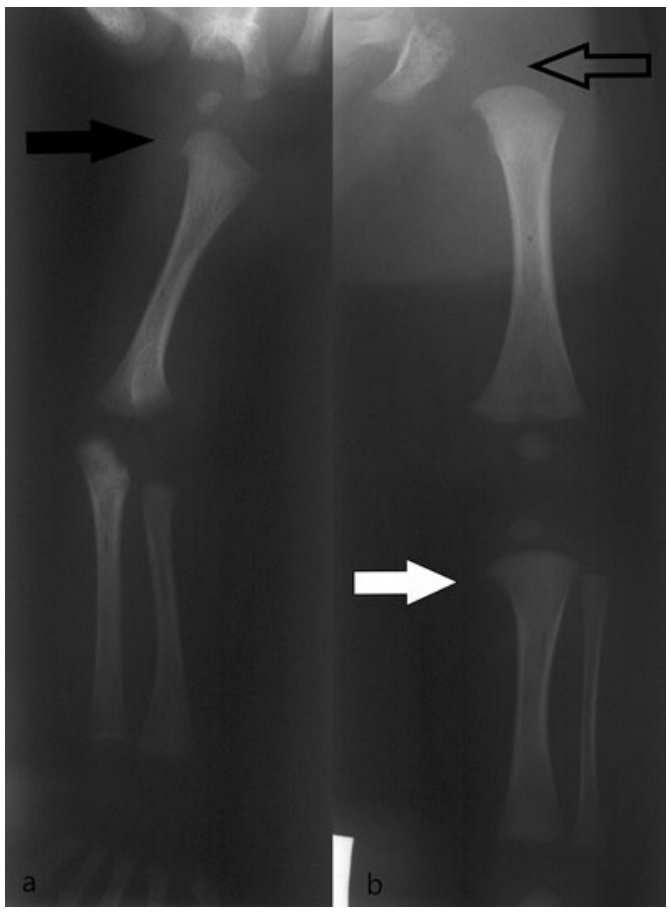

Fig. 2. Radiogram of the upper and lower extremities in a new-born girl with Jeune syndrome: general limb shortening and rhizomelia, but without the typical precocious proximal femoral epiphyseal ossification in this case (from our own archives).

recommended to image previously normal sites unless clinically indicated. In fact, some radiographic abnormalities may only become visible at an older age, making serial radiographic evaluation necessary (3). Recently, Watson at el. proposed the introduction of a standardised protocol that may be adapted in certain specific situations (9). They found that due to the variability of imaging protocols used at different centres, radiographs may be inadequate or incomplete, resulting in diagnostic delays. The proposed standardised protocol could optimize images, particularly if referred for an expert opinion, reduce the radiation dose from unnecessary exposure, and enable faster diagnosis. The radiographs should be of the high quality necessary for precise analysis. They also recommended common indications for timing of skeletal dysplasia imaging. 


\begin{tabular}{l} 
Table 1. A systematic approach: ABC evaluation of suspected skeletal dysplasia as proposed by Offiah and \\
Hall (10) \\
\begin{tabular}{ll}
\hline A - Anatomical location/ alignment \\
\hline Axial skeleton & Skull and Spine, Ribs and Pelvis \\
\hline Appendicular skeleton & Shortening (Rhizomelic, Mesomelic, Acromelic, Micromelic) \\
\cline { 2 - 2 } & Location (Epiphyseal, Metaphyseal, Diaphyseal) \\
\hline B - Bones (5 S) & \\
\hline Structure, Shape, Size, Sum, Soft tissue. \\
\hline C - Complications
\end{tabular} \\
\hline Fractures, Atlanto-axial subluxation, Progressive scoliosis, Limb length discrepancies, Malignancy, Complication of therapy \\
\hline D - Dead/Alive
\end{tabular}

The interpretation of the skeletal survey is a challenge for paediatric radiologists with an untrained eye for skeletal disorders. The radiologist needs to analyse the skeletal survey radiographs in a systematic way, and without an organized approach the task may seem overwhelming. Two main approaches, Offiah's and Alany's, are suggested in order not to miss important details regarding the morphological or structural features of the bones (10-12). Offiah and Hall suggested the ABC evaluation of suspected $\mathrm{SD}$, which is easy to remember and easy to use (Table1) (10).

"A" describes the anatomical localisation and alignment. The axial skeleton is often involved and manifests as a short spine, either due to a generalised reduction in the vertebral body (platyspondyly) or due to an angular deformity of the spine (scoliosis, kyphosis). When the limbs (appendicular skeleton) are involved, the type of bone shortening should be determined, as well as the predominant location of the morphological changes. Predominant involvement of the epiphyses might lead to premature osteoarthritis, of the diaphysis to deformities of the affected bones, and of the metaphysis to short bones. Descriptions of the bone alignment include dislocation, subluxation, scoliosis and kyphosis.

"B" describes different features of the individual bone with five "S": structure (focused on bone density, but also include a description of wormian bones, exostoses, enchon- dromata), shape (using descriptive radiological terms such as angel-shaped, cone-shaped, stipples, flared, scalloped, trident, etc.), size (larger, smaller), sum (too few or too many bones, fused, absent or with delayed ossification), and soft tissues (wasted or excessive, contractures, calcification).

"C" describes the complications which could be part of a condition (mostly pathological fractures, compression of adjacent structures, malalignment, malignant alteration) or secondary to treatment.

" $\mathrm{D}$ " describes dead or alive. If the SD is lethal, this might help in diagnosis (affect the subtype) and even change the mode of inheritance.

On the other hand, Alanay et al. suggested three steps of assessment, which are similar, but less precise than the $\mathrm{ABC}$ evaluation (11, 12). As the first step, assessment of the disproportion is performed (a quick look at the spine - short trunk and at the extremities may help to define rhizomelia, mesomelia, and acromelia). The second step is assessment of epiphyseal, metaphyseal, and diaphyseal ossification. The third step, is the differentiation of normal variants from pathological abnormalities by an experienced paediatric radiologist.

We should be aware that sometimes no specific, firm radiological diagnosis can be reached. No diagnosis is better than a wrong diagnosis, particularly if it leads to inappropriate and expensive genetic testing, and inap- 
propriate genetic counselling (1). Veeramani et al. (13) found that a clear diagnosis of SD is not possible in a third of cases (skeletal abnormalities were presented but a clear diagnosis could not be reached). In addition, their research suggested that there are differences in diagnosis rates for patients who have had a full skeletal survey compared to a limited survey $(79 \%$ v. $44 \%)$. There is a need for widely accepted standardization of skeletal surveys and for access to multidisciplinary, highly specialized teams (13). Expert opinion may be sought either face-to-face (multidisciplinary meetings) or via web-based consultation (the European Skeletal Dysplasia Network or the International Skeletal Dysplasia Society).

\section{Conclusion}

SDs are not as uncommon as once thought. Without doubt, the diagnosis and management of SDs needs teamwork and multidisciplinary management, including paediatric (endocrine) radiologists, geneticists, and orthopaedists. A full skeletal survey should be the first line of investigation when a SD is suspected, followed by targeted molecular, biochemical and genetic tests, based on the radiological and clinical findings.

Conflict of interest: The author declares that she has no conflict of interest.

\section{References}

1. Offiah AC. Skeletal dysplasias: An overview. In: Allgove J Shaw NJ (eds): Calcium and Bone Disorders in children and adolescents. 2nd, revised edition. Endocr Dev. Basel: Karger; 2015;28:259-76.
2. Panada A, Gamanagatti S, Jana M, Kumar Gupta A. Skeletal dysplasias: A radiographic approach and review of common non-lethal skeletal dysplasias. World J Radiol. 2014;6(10):808-25.

3. Mortier GR. The diagnosis of skeletal dysplasias: a multidisciplinary approach. Eur J Radiol. 2001;40:161-7.

4. Spranger J. Pattern recognition in bone dysplasias. Prog Clin Biol Res. 1985;200:315-42.

5. Spranger J. Bone dysplasia 'families'. Pathol Immunopathol Res. 1988;7:76-80.

6. Bonafe L, Cormier-Daire V, Hall C, Lachman R, Mortier G, Mundlos S, et al. Nosology and classification of genetic skeletal disorders: 2015 revision. Am J Med Genet A. 2015;167A(12):2869-92.

7. Kant SG, Grote F, de Ru MH, Oostdijk W, Zonderland HM, Breuning $\mathrm{MH}$, et al. Radiographic evaluation of children with growth disorders. Horm Res. 2007;68:310-5.

8. Parnell SE, Phillips GS. Neonatal skeletal dysplasias. Pediatr Radiol. 2012;42(Suppl 1):S150-S157.

9. Watson SG, Calder AD, Offiah AC, Negus S. A review of imaging protocols for suspected skeletal dysplasia and proposal for standardisation. Pediat Radiol. 2015;45:1773-7.

10. Offiah AC, Hall CM. Radiological diagnosis of the constitutional disorders of bone. As easy as A, B, C? Pediatr Radiol. 2003;33:153-61.

11. Alanay Y, Lachmann RS. A review of the principles of radiological assessment of skeletal dysplasias. J Clin Res Pediatr Endocrinol. 2011;3(4):163-78.

12. Lachmann RS. Skeletal dysplasias. In Slovis TL, ed. Caffey's Pediatric diagnostic imaging. 11th ed. Philadelphia: Mosby Elsevier; 2008:2613-725.

13. Veeramani AKI, Higgins P, Butler S, Donaldson M, Dougan E, Duncan R, et al. Diagnostic use of skeletal survey in suspected skeletal dysplasia. J Clin Res Pediatr Endocrinol. 2009;1(6):270-4. 